TITLE:

\title{
Improved Production of Plant Isoquinoline Alkaloids by Metabolic Engineering
}

$\operatorname{AUTHOR}(\mathrm{S})$ :

Sato, Fumihiko

CITATION:

Sato, Fumihiko. Improved Production of Plant Isoquinoline Alkaloids by Metabolic Engineering. Advances in Botanical Research 2013, 68: 163181

ISSUE DATE:

2013

URL:

http://hdl.handle.net/2433/178035

RIGHT:

C 2013 Elsevier Ltd.; この論文は出版社版でありません。引用の際には 出版社版をご確認ご利用ください。; This is not the published version. Please cite only the published version. 


\title{
Improved Production of Plant Isoquinoline Alkaloids by Metabolic Engineering
}

\author{
Fumihiko Sato
}

Division of Integrated Life Science, Graduate School of Biostudies, Kyoto University, Oiwake-cho, Kitashirakawa, Sakyo-ku, Kyoto 606-8502, Japan; email: fsato@lif.kyoto-u.ac.jp

\section{Contents}

1. Introduction

2. Isoquinoline alkaloid biosynthesis and pathway characterization

3. Metabolic pathway engineering

3.1. Overexpression of the rate-limiting enzyme and transcription factors for yield improvement

3.2. Trimming of pathways, and the introduction of new branch pathways for quality improvement

4. Perspectives

5. Conclusions

Acknowledgements

References 


\begin{abstract}
Higher plants produce diverse low-molecular-weight chemicals such as alkaloids, terpenoids and phenylpropanoid compounds. Among these chemicals, alkaloids are particularly important in medicine due to their high biological activities. However, the low yield of metabolites, especially alkaloids, in plants limits large-scale development of the plant natural product industry. In this chapter, I describe the metabolic engineering of plants to improve the yield and quality of useful secondary metabolites and their potentials. Among alkaloids, I focus on the production of isoquinoline alkaloids (IQAs), since their biosynthesis has been most intensively studied at the molecular level and their application in metabolic engineering has also been intensively examined. Based on our understanding of IQA engineering, I discuss its application to the production of other alkaloids through similar approaches.
\end{abstract}

Keywords: isoquinoline alkaloid ' metabolic engineering $\cdot$ overexpression of rate-limiting enzyme $\cdot \mathrm{RNAi} \cdot$ transcription factor
Abbreviations
$\mathrm{BBE}$, berberine bridge enzyme;
Cj, Coptis japonica;
CNMT, coclaurine $N$-methyltransferase;
CoOMT, columbamine $O$-methyltransferase;
$\mathrm{COR}$, codeinone reductase;
CYP80A1, berbamunine synthase;
CYP80B1, $N$-methylcoclaurine 3'-hydroxylase;
CYP82G2, corytuberine synthase;
CYP82N2v2 (P6H), protopine 6-hydroxylase;
CYP719A1, canadine synthase;
CYP719A5, chelanthifoline synthase;
CYP719A2/A3, stylopine synthase;
CYP719B1, salutaridine synthase;
IQAs, isoquinoline alkaloids;
MeJA, methyl jasmonate;
NCS, norcoclaurine synthase;
OMT, $O$-methyltransferases;
6OMT, norcoclaurine 6OMT; 
4'OMT, 3'-hydroxy- $N$-methyl-coclaurine 4'OMT;

RNAi, RNA interference;

SalAT, salutaridinol-7- $O$-acetyltransferase;

SalR, salutaridine reductase;

SMT, $(S)$-scoulerine 9-O-methyltransferase;

THBO, tetrahydroprotoberberine oxidase 


\section{Introduction}

Higher plants produce diverse low-molecular-weight chemicals such as alkaloids, terpenoids and phenylpropanoid compounds (Croteau et al., 2000). Although these compounds are widely used for health and nutrition in humans, alkaloids are particularly important in medicine due to their high biological activities. However, the low yield of metabolites, especially alkaloids, in plants limits large-scale development of the plant natural product industry. Thus, many research groups have tried to establish production systems using the micropropagation of elite plant clones, cell culture and organ culture based on incomplete information regarding biosynthesis of these metabolites. Over the past 20 years, our understanding of the biochemistry and molecular biology of natural products, including alkaloids, has rapidly expanded. Based on knowledge regarding biosynthetic enzymes and their corresponding genes, the modification of productivity and metabolic profiles has become possible (Sato et al., 2007a; Sato \& Yamada, 2008). Furthermore, the microbial production of plant metabolites through the reconstruction of plant biosynthetic pathways has become feasible (Chow \& Sato, 2013; Hawkins \& Smolke, 2008; Minami et al., 2008; Nakagawa et al., 2011; see also Chapter 7). Metabolic engineering in plant cells has advantages and disadvantages. In this chapter, I discuss its potentials and pitfalls based on our experience.

\section{Isoquinoline alkaloid biosynthesis and pathway characterization}

Due to technological difficulties in the cultivation and relatively slow growth of medicinal plants, plant cell and tissue cultures have been intensively examined for the production of secondary metabolites (Sato \& Yamada, 2008; Verpoorte et al., 1991). Whereas the selection of high-yield lines and treatment with an elicitor such as methyl jasmonate has enabled the industrial production of some metabolites, e.g., shikonin-production in selected Lithospermum erythrorhizon cells, or paclitaxel production in Pacific yew cell cultures with methyl jasmonate, it is quite difficult to achieve both high productivity and stability for industrial application (Sato \& Yamada, 2008). While the development of hairy roots by the transformation of plant cells with Agrobacterium rhizogenes provides a solution with unlimited growth, morphological differentiation of roots for physical strength, high potential for the mass-production of valuable secondary metabolites, and greater genetic stability, for fermentation, we need a much faster growth of cells/tissues, higher productivity and improved quality of 
metabolites (for details of cell culture techniques, please see Sato \& Yamada, 2008).

Recent advances in molecular biology have provided tools for metabolic engineering. The identification of many genes of biosynthetic enzymes and characterization of the spatial and developmental regulation of their expression have clarified their roles in the biosynthesis of secondary metabolites and provided strategies for metabolic engineering such as the trimming of undesired pathways, the enhancement of rate-limiting steps and the introduction of new pathways to produce novel compounds (Sato et al., 2007a,b).

Isoquinoline alkaloid (IQA) biosynthesis provides a good model in metabolic engineering, since it has been intensively studied at the molecular level and experimentally challenged. The biosynthetic pathway for IQAs starts with L-tyrosine, as shown in Figure 1. Among IQAs, the biosynthesis of benzylisoquinoline alkaloid from norcoclaurine has been the most investigated to date. All of the enzyme genes of the total nine enzymatic reaction steps from norcoclaurine to berberine have been characterized at the DNA level (Fig. 1): a norcoclaurine synthase (NCS; an entry enzyme in IQA biosynthesis, PR10 family such as TfNCS from Thalictrum flavum (Lee \& Facchini, 2010; Samanani et al., 2004) and a novel dioxygenase-like protein, CjNCS1, from Coptis japonica (Minami et al., 2007)), an $N$-methyltransferase (coclaurine $N$-methyltransferase (CNMT, Choi et al., 2002)), three $O$-methyltransferases (OMTs; norcoclaurine 6OMT, 3'-hydroxy- $N$-methyl-coclaurine 4'OMT (6OMT, 4'OMT, Morishige et al., 2000), (S)-scoulerine 9-O-methyltransferase (SMT, Takeshita et al., 1995), a hydroxylase (CYP80B1, Pauli \& Kutchan, 1998), a berberine bridge enzyme (BBE, Dittrich \& Kutchan, 1991), a methylenedioxy ring-forming enzyme (canadine synthase; CYP719A1, Ikezawa et al., 2003), and a tetrahydroprotoberberine oxidase (THBO, Gesell et al., 2011; Matsushima et al., 2012).

>>Insert Figure 1 here

The number of available enzyme genes is growing rapidly (Figs. 1 and 2): the genes of corytuberine synthase in aporphine biosynthesis (CYP82G2, Ikezawa et al., 2008); those of salutaridine synthase (CYP719B1, Gesell et al., 2009), salutaridine reductase (SalR, Ziegler et al., 2006), salutaridinol-7-O-acetyltransferase (SalAT, Grothe et al., 2001), thebaine 6- $O$-demethylase and codeine $O$-demethylase (T6ODM, CODM, Hagel \& Facchini, 2010) and NADPH-dependent codeinone reductase (COR, Unterlinner et al., 1999) in morphinan alkaloid biosynthesis; those of chelanthifoline synthase (CYP719A5, Ikezawa et al., 2009), stylopine synthase (CYP719A2/A3, 
Ikezawa et al., 2007), (S)-cis- $N$-methylstylopine 14-hydroxylase (Beaudoin \& Facchini, 2013), protopine 6-hydroxylase (CYP82N2v2, Takemura et al., 2013), sanguinarine reductase (Vogel et al., 2010) and dihydrobenzophenanthridine oxidase (DBOX, Hagel et al., 2012) in benzophenanthridine biosynthesis; those of berbamunine synthase (CYP80A1, Kraus \& Kutchan, 1995) in the bis-benzylisoquinoline alkaloid pathway; those of $O$-methyltransferases (Dang \& Facchini, 2012) and a 10-gene cluster (three $O$-methyltransferases (PSMT1-3), four cytochrome P450s (CYP82X1/2, CYP82Y1 \& CYP719A21), an acetyltransferase (PSAT1), a carboxylesterase (PSCXE1) and a short-chain dehydrogenase/reductase (PSSDR1)(Winzer et al., 2012) in noscapine biosynthesis and those of $O$-methyltransferases in emetine biosynthesis (IpeOMT1/2/3, CiOMT, Cheong et al., 2011; Nomura \& Kutchan, 2010).

>>Insert Figure 2 here

While many homologues are annotated after cloning of the first enzyme gene, we should pay close attention to the properties of each enzyme, since pathways in secondary metabolism are often diversified and connected in a complicated manner, based on the different substrate and reaction specificity of the enzyme acquired during the evolution of each species. For example, while Coptis japonica columbamine $O$-methyltransferase $(C j \mathrm{CoOMT})$ can use both tetrahydrocolumbamine and columbamine in palmatine biosynthesis, columbamine was once thought to be the exclusive substrate of CoOMT in Berberis plants (Morishige et al., 2002). Similarly, CNMT of Coptis and Berberis showed different substrate specificities; i.e., the Coptis enzyme could $N$-methylate norlaudanosoline, while the Berberis enzyme could not. In this sense, the knowledge needed for both metabolic engineering and synthetic biology is still incomplete, and such information is greatly needed. We should be careful in the annotation of an enzyme gene simply based on homology, since only a few changes in amino acids can modify reaction specificities; e.g., Ipecac OMTs in emetine biosynthesis (Fig. 3; Cheong et al., 2011; Nomura \& Kutchan, 2010).

>> Insert Figure 3 here

\section{Metabolic pathway engineering}

3.1. Pathway engineering with overexpression of the rate-limiting enzyme and transcription factors in alkaloid biosynthesis 
With an increase in the molecular tools available in metabolic engineering, we can now modulate the metabolic flow of plant cells. Among several strategies, overexpression of the gene for the enzyme in the rate-limiting step is commonly examined to improve the yield (Fig. 4). The overexpression of $C$. japonica SMT in the original cells was the first successful application of genetic engineering to IQA biosynthesis to increase berberine production (Sato et al., 2001), while 4'OMT overexpression was later successfully applied in intact plants (Inui et al., 2012). Allen et al. (2008) also reported that transgenic opium poppy with overexpression of SalAT had more than $30 \%$ greater total alkaloids than the control in three independent trials over 3 years.

However, overexpression of an endogeneous gene can (often) induce the co-suppression of the gene and decrease the production of the end-metabolites (Jorgensen, 1995; Takemura et al., 2010a). Thus, heterologous expression of a homologue isolated from a different plant species is recommended. For example, Cj6OMT was expressed in California poppy (Eschschlozia californica) cells to overcome the rate-limiting step (Inui et al., 2007). Note that bottleneck step(s) may differ among plant species and cell lines due to variations in gene expression. In fact, the overexpression of $C j 4$ 'OMT was less effective for increasing alkaloid production than that of 6OMT in E. californica cells, whereas 4'OMT was effective in $C$. japonica plants (Inui et al., 2007, 2012). Changes in the bottleneck step may also occur after modification of the enzyme expression level through metabolic engineering.

Since there are multiple bottleneck steps and these limiting steps can change with overexpression of the enzyme in the limiting step, overall regulation of the expression levels of biosynthetic genes in the pathway is the preferred strategy. Whereas information about the transcription factors in alkaloid biosynthesis is very limited, several transcription factors, such as the ERF, WRKY and bHLH families in alkaloid biosynthesis, including IQA biosynthesis, have been isolated and characterized (Kato et al., 2007; Yamada et al., 2011a,b; Yamada \& Sato, 2013). While experimental results suggest that the activities of native transcription factors can be fine-tuned spatially and temporally (see Yamada \& Sato, 2013), the successful application of the overexpression of heterologous $A t \mathrm{WRKY}$ transcription factor in opium poppy to increase the morphinan alkaloid content (Apuya et al., 2008) suggests that transcription factors may be used to improve the yields of IQAs and other alkaloids.

>>Insert Figure 4 here 


\subsection{Pathway engineering with trimming of pathways and the introduction of new branch pathways}

While an increase in the metabolite yield is important for industrial application, modification of the metabolite profile by the introduction of a new metabolic branching point and/or the trimming of undesired pathways is another important approach in drugs development.

To increase metabolite diversity, we ectopically expressed CjSMT, which is involved in berberine biosynthesis but not in benzophenanthridine alkaloid biosynthesis, in California poppy (E. californica) cells (Fig. 5). CjSMT expression not only produced columbamine (oxidized product of tetrahydrocolumbamine) in transgenic E. californica cells (Sato et al., 2001) but also induced the accumulation of novel products, i.e., allocryptopine and 10-hydroxychelerythrine, derived from $C j$ SMT reaction products by the endogenous enzyme reactions in E. californica cells, indicating that the alkaloid profile in transgenic cells was further diversified by the introduction of a branched pathway (Takemura et al., 2010b).

>> Insert Figure 5 here

While an increase in metabolite diversity is preferable for the screening of novel products, the reduction of undesired metabolic diversity is also required to prepare a single purified chemical and increase the metabolic flow to the desired compound. Among the methods for gene silencing (e.g., antisense, RNA silencing, knockout with T-DNA insertion, mutation,.. etc.), RNA silencing with double-stranded RNA molecules (RNA interference; RNAi with short interfering (si) RNA or micro (mi) RNA, co-suppression of gene expression with overexpression of a target gene, or virus-induced gene silencing (VIGS)) is the most powerful and reliable, although Transcription Activator-Like Effector Nucleases (TALEN) could be useful for preparing knockout mutant and novel mutants through homologous recombinants (Borgio, 2009; Desgagné-Penix \& Facchini, 2012; Sato, 2005; Winzer et al., 2012; Zhang et al., 2013).

Successful RNAi of BBE in California poppy cells induced the accumulation of reticuline, a substrate of BBE (Fujii et al., 2007), whereas antisense RNA of BBE produced little accumulation of reticuline in transgenic cells (Park et al., 2002) (Fig. 6). However, there is another pitfall in effective gene silencing; e.g., an enhanced branch pathway from the accumulated reticuline to produce a byproduct, i.e., 7-O-methylreticuline, which could scarcely be detected in control cells. In addition, 
suppression of a certain enzyme in a metabolic pathway may induce the unexpected disturbance of the pathway; e.g., RNAi of COR genes in opium poppy induced the accumulation of a far-upstream precursor, (S)-reticuline (Allen et al., 2004). The disturbance of metabolism by the down-regulation of enzyme expression can be partly explained by the accumulation of an intermediate and feedback regulation of the pathway, or the disruption of an enzyme complex (metabolome) to catalyze sequential reactions, or off-target effects of RNAi on other unexpected gene targets (Allen et al., 2004, 2008).

\section{>> Insert Figure 6 here}

While simple overexpression of the rate-limiting step enzyme/transcription factors, introduction of a branch pathway and trimming of a pathway through the use of gene silencing are currently applied in metabolic engineering in alkaloid biosynthesis, the combination of these strategies provides a more effective approach for improving both the quantity and quality of metabolites. For example, trimming of the endogenous pathway with the introduction of new branch was critical for drastic modification of the metabolite in blue rose production; i.e., the accumulation of sufficient blue pigment, delphinidin, was only achieved by the combined expression of the gene for a novel blue pigment biosynthetic enzyme, i.e., flavonoid 3',5'-hydroxylase, and the down-regulation of endogenous dihydroflavonol 4-reductase by RNAi to shut down the undesired red pigment pathway (Katsumoto et al., 2007). Such a combined approach can be performed with a transcription factor and an enzyme for a rate-limiting step that is not under the control of the introduced transcription factor. This can provide greater flexibility in metabolic engineering.

\section{Perspectives}

While the biosynthetic pathways for many metabolites still require considerable characterization including of the involved enzymes and their genes, continued advances in molecular and genomic techniques will surely expand the production of useful natural products, especially alkaloids. Particularly, whole genome sequence data bring considerable information on biosynthesis along with rapidly accumulating RNA sequence data. The combined analysis of transcriptomes, proteomes, metabolomes and genomes provides a new approach for identifying the candidate genes in uncharacterized pathways (Dang \& Facchini, 2012; Desgagné-Penix et al., 2010, 2012; Farrow et al., 2012; Takemura et al., 2010b; Winzer et al., 2012). The 
establishment of transcriptome and proteome databases for the investigation of natural product metabolism in non-model plant systems has provided useful information for metabolic engineering. Desgagné-Penix et al. (2010) showed that profiling of the more-abundant proteins in elicitor-treated opium poppy cell cultures revealed several uncharacterized enzymes that potentially catalyze steps in sanguinarine biosynthesis.

Structural biology also provides important cues for isolating key biosynthetic enzymes in pathways and their molecular functions, although structural information regarding the biosynthetic enzymes in secondary metabolism is very limited (Ilari et al., 2009; Wallner et al., 2012; Winkler et al., 2009).

Whereas we can use both plant and microbial cells as a platform for metabolite production (Chow \& Sato, 2013; see also Chapter 7), we should be aware of their pros and cons. For instance, while plant cell or tissue cultures can produce more divergent chemicals than microbial systems, their complexity and instability are problematic for industrial production. Microbial systems enable alkaloid production on a relatively large scale within a short period, but require more molecular informations on the biosynthetic pathways and enzymes, whereas the emergence of synthetic biology offers the possibility of improving the production of useful products through the design and engineering of complex biological systems. While computer-assisted molecular design software is being developed to facilitate the synthesis of novel compounds with drug-like properties and desired activities, the use of information science for the reconstruction of metabolic pathways, i.e., "retrobiosynthetic" systems, will require more experimental data on the identification and analysis of biosynthetic enzymes and pathways. However, technological progress has been quite rapid and provides us with many options. The potentials of metabolic engineering in secondary metabolism are unlimited.

\section{Conclusion}

Metabolic engineering as well as synthetic biological approaches are at the forefront in natural product research. Our experiences in IQA biosynthesis provide new clues for the molecular characterization of biosynthetic pathways and novel production systems for natural products. Technological advances, including next-generation sequencing, offer a bright future for the production of not only IQAs but also other alkaloids. This high potential in natural product research will require more sophisticated techniques as we move from metabolites to drug discovery.

\section{Acknowledgement}


This research was supported by the Ministry of Education, Culture, Sports, Science and Technology of Japan [Grant-in-Aid (No. 21248013 to F. S.)].

\section{References}

1. Allen, R.S., Miller, J.A.C., Chitty J.A., Fist A.J., Gerlach W.L., \& Larkin P.J, (2008). Metabolic engineering of morphinan alkaloids by overexpression and RNAi suppression of salutaridinol 7-O-acetyltransferase in opium poppy. Plant Biotechnology Journal, 6, 22-30.

2. Allen, R.S., Millgate, A.G., Chitty, J.A., Thistleton, J., Miller, J.A.C., Fist, A.J., et al. (2004). RNAi-mediated replacement of morphine with the non narcotic alkaloid reticuline in opium poppy. Nature Biotechnology, 22, 1559-1566.

3. Apuya, N.R., Park, J.H., Zhang, L., Ahyow, M., Davidow, P., Van Fleet, J., et al. (2008). Enhancement of alkaloid production in opium and California poppy by transactivation using heterologous regulatory factors. Plant Biotechnology Journal, 6, 160-175.

4. Beaudoin, G.A., \& Facchini, P.J. (2013). Isolation and characterization of a cDNA encoding (S)-cis- $N$-methylstylopine 14-hydroxylase from opium poppy, a key enzyme in sanguinarine biosynthesis. Biochemical and Biophysical Research Communications, 431, 597-603.

5. Borgio, J. (2009). RNA interference (RNAi) technology: a promising tool for medicinal plant research. Journal Medicinal Plant Research, 3, 1176-1183.

6. Cheong, B.E., Takemura, T., Yoshimatsu, K., \& Sato, F. (2011). Molecular cloning of an $O$-methyltransferase from adventitious roots of Carapichea ipecacuanha. Bioscience Biotechnology Biochemistry, 75, 107-113.

7. Choi, K.B., Morishige, T., Shitan, N., Yazaki, K., \& Sato, F. (2002) Molecular cloning and characterization of coclaurine $N$-methyltransferase from cultured cells of Coptis japonica. The Journal of Biological Chemistry, 277, 830-835.

8. Chow, Y.L., \& Sato, F. (2013) Metabolic engineering and synthetic biology for the production of isoquinoline alkaloids. In: S. Chandra, H. Lata H., \& A. Varma (Eds), Biotechnology for Medicinal Plants: Micropropagation and Improvement (pp. 327-343). Berlin, Springer.

9. Croteau, R., Kutchan, T.M., \& Lewis, N.G. (2000). Natural products (Secondary metabolites). In: B.B. Buchanan, W. Gruissem, \& R.L. Jones (Eds), Biochemistry \& Molecular Biology of Plants (pp. 1250-1318). Maryland, American Society of Plant Biologists.

10. Dang, T.T., \& Facchini, P.J. (2012). Characterization of three O-methyltransferases 
involved in noscapine biosynthesis in opium poppy. Plant Physiology, 159, 618-631.

11. Desgagné-Penix, I., Khan, M.F., Schriemer, D.C., Cram, D., Nowak, J., \& Facchini, P.J. (2010). Integration of deep transcriptome and proteome analyses reveals the components of alkaloid metabolism in opium poppy cell cultures. BMC Plant Biology, 10, 252-268.

12. Desgagné-Penix, I., \& Facchini, P.J. (2012) Systematic silencing of benzylisoquinoline alkaloid biosynthetic genes reveals the major route to papaverine in opium poppy. The Plant Journal, 72, 331-344.

13. Desgagné-Penix, I., Farrow, S.C., Cram, D., Nowak, J., \& Facchini, P.J. (2012). Integration of deep transcript and targeted metabolite profiles for eight cultivars of opium poppy. Plant Molecular Biology, 79, 295-313.

14. Dittrich, H., \& Kutchan, T.M. (1991). Molecular cloning, expression, and induction of berberine bridge enzyme, an enzyme essential to the formation of benzophenanthridine alkaloids in the response of plants to pathogenic attack. Proceedings of the National Academy of Sciences of the United States of America, 88, 9969-9973.

15. Facchini, P.J., Huber-Allanach, K.L. \& Tari, L.W. (2000). Plant aromatic L-amino acid decarboxylases: evolution, biochemistry, regulation, and metabolic engineering applications. Phytochemistry, 54, 121-138.

16. Farrow, S.C., Hagel, J.M., \& Facchini, P.J. (2012). Transcript and metabolite profiling in cell cultures of 18 plant species that produce benzylisoquinoline alkaloids. Phytochemistry, 77, 79-88.

17. Fujii, N., Inui, T., Iwasa, K., Morishige, T., \& Sato, F. (2007). Knockdown of berberine bridge enzyme by RNAi accumulates $(S)$-reticuline and activates a silent pathway in cultured California poppy cells. Transgenic Research, 16, 363-375.

18. Gesell, A., Rolf, M., Ziegler, J., Díaz Chávez, M.L., Huang, F.-C., \& Kutchan T.M. (2009). CYP719B1 is salutaridine synthase, the C-C phenol-coupling enzyme of morphine biosynthesis in opium poppy. The Journal of Biological Chemistry, 284, $24432-24442$.

19. Gesell, A., Díaz Chávez, M.L., Kramell, R., Piotrowski, M., Macheroux, P., \& Kutchan, T.M. (2011). Heterologous expression of two FAD-dependent oxidases with $(S)$-tetrahydroprotoberberine oxidase activity from Argemone mexicana and Berberis wilsoniae in insect cells. Planta 233, 1185-1197.

20. Grothe, T., Lenz, R., \& Kutchan, T.M. (2001). Molecular characterization of the salutaridinol-7-O-acetyltransferase involved in morphine biosynthesis in opium 
poppy Papaver somniferum. Journal of Biological Chemistry, 276, 30717-30723.

21. Hagel, J.M., \& Facchini, P.J. (2010). Dioxygenases catalyze the $O$-demethylation steps of morphine biosynthesis in opium poppy. Nature Chemical Biology, 6, 273-275.

22. Hagel, J.M., Beaudoin, G.A., Fossati, E., Ekins, A., Martin, V.J., \& Facchini, P.J. (2012). Characterization of a flavoprotein oxidase from opium poppy catalyzing the final steps in sanguinarine and papaverine biosynthesis. The Journal of Biological Chemistry, 287, 42972-42983.

23. Hawkins, K., \& Smolke, C. (2008). Production of benzylisoquinoline alkaloids in Saccharomyces cerevisiae. Nature Chemical Biology, 4, 564-573.

24. Ikezawa, N., Tanaka, M., Nagayoshi, M., Shinkyo, R., Sakaki, T., Inouye, K., et al. (2003). Molecular cloning and characterization of CYP719, a methylenedioxy bridge-forming enzyme that belongs to a novel P450 family, from cultured Coptis japonica cells. The Journal of Biological Chemistry, 278, 38557-38565.

25. Ikezawa, N., Iwasa, K., \& Sato, F. (2007). Molecular cloning and characterization of methylenedioxy bridge-forming enzymes involved in stylopine biosynthesis in Eschscholzia californica. FEBS Journal, 274, 1019-1035.

26. Ikezawa, N., Iwasa, K., \& Sato, F. (2008). Molecular cloning and characterization of CYP80G2, a cytochrome P450 that catalyzes an intramolecular $\mathrm{C}-\mathrm{C}$ phenol coupling of $(S)$-reticuline in magnoflorine biosynthesis, from cultured Coptis japonica cells. The Journal of Biological Chemistry, 283, 8810-8821.

27. Ikezawa, N., Iwasa, K., \& Sato, F. (2009). CYP719A subfamily of cytochrome p450 oxygenase and isoquinoline alkaloid biosynthesis in Eschscholzia californica. Plant Cell Reports, 28, 123-133.

28. Ilari, A., Franceschini, S., Bonamore, A., Arenghi, F., Botta, B., Macone, A. et al. (2009). Structural basis of enzymatic (S)-norcoclaurine biosynthesis. The Journal of Biological Chemistry, 284, 897-904

29. Inui, T., Tamura, K., Fujii, N., Morishige, T., \& Sato, F. (2007). Overexpression of Coptis japonica norcoclaurine 6-O-methyltransferase overcomes the rate-limiting step in benzylisoquinoline alkaloid biosynthesis in cultured Eschscholzia californica. Plant and Cell Physiology, 48, 252-262.

30. Inui, T., Kawano, N., Shitan, N., Yazaki, K., Kiuchi, F., Kawahara, N., et al. (2012). Improvement of benzylisoquinoline alkaloid productivity by overexpression of 3'-hydroxy- $N$-methylcoclaurine 4'- $O$-methyltransferase in transgenic Coptis japonica plants. Biological \& Pharmaceutical Bulletin, 35, 650-659.

31. Jorgensen, R.A. (1995). Cosuppression, flower color patterns, and metastable gene 
expression states. Science, 268, 686-691.

32. Kato, N., Dubouzet, E., Kokabu, Y., Yoshida, S., Taniguchi, Y., Dubouzet, J.G., et al. (2007). Identification of a WRKY protein as a transcriptional regulator of benzylisoquinoline alkaloid biosynthesis in Coptis japonica. Plant and Cell Physiology, 48, 8-18.

33. Katsumoto, Y., Fukuchi-Mizutani, M., Fukui, Y., Brugliera, F., Holton, T.A., Karan, M., et al. (2007). Engineering of the rose flavonoid biosynthetic pathway successfully generated blue-hued flowers accumulating delphinidin. Plant and Cell Physiology, 48, 1589-1600.

34. Kraus, P.F., \& Kutchan, T.M. (1995). Molecular-cloning and heterologous expression of a cDNA-encoding berbamunine synthase, a C-O-phenol-coupling cytochrome-P450 from the higher-plant Berberis stolonifera. Proceedings of the National Academy of Sciences of the United States of America, 92, 2071-2075.

35. Lee, E.J., \& Facchini, P. (2010). Norcoclaurine synthase is a member of the pathogenesis-related 10/Bet v1 protein family. The Plant Cell, 22, 3489-3503.

36. Lee, E.J., \& Facchini, P.J. (2011). Tyrosine aminotransferase contributes to benzylisoquinoline alkaloid biosynthesis in opium poppy. Plant Physiology, 157, 1067-1078.

37. Matsushima, Y., Minami, H., Hori K., \& Sato F. (2012). Pathway engineering of benzylisoquinoline alkaloid biosynthesis in transgenic California poppy cells with ectopic expression of tetrahydroberberine oxidase from Coptis japonica. Plant Biotechnology, 29, 473-481.

38. Minami, H., Dubouzet, E., Iwasa, K., \& Sato, F. (2007). Functional analysis of norcoclaurine synthase in Coptis japonica. The Journal of Biological Chemistry, 282, 6274-6282.

39. Minami, H., Kim, J.-S., Ikezawa, N., Takemura, T., Katayama, T., Kumagai, H., et al. (2008). Microbial production of plant benzylisoquinoline alkaloids. Proceedings of the National Academy of Sciences of the United States of America, 105, 7393-7398.

40. Morishige, T., Tsujita, T., Yamada, Y., \& Sato, F. (2000). Molecular characterization of the $S$-adenosyl-L-methionine: 3'-hydroxy- $N$-methylcoclaurine 4'-O-methyltransferase involved in isoquinoline alkaloid biosynthesis in Coptis japonica. The Journal of Biological Chemistry, 275, 23398-23405.

41. Morishige, T., Dubouzet, E., Choi, K.-B., Yazaki, K., \& Sato, F. (2002). Molecular cloning of columbamine $O$-methyltransferase from cultured Coptis japonica cells. European Journal of Biochemistry, 269, 5659-5667. 
42. Nakagawa, A., Minami, H., Kim, J.S., Koyanagi, T., Katayama, T., Sato, F., et al. (2011). A bacterial platform for fermentative production of plant alkaloids. Nature Communications 2 Article number: 326, doi:10.1038/ncomms1327.

43. Nomura, T., \& Kutchan, T.M. (2010). Three new $O$-methyltransferases are sufficient for all $O$-methylation reactions of Ipecac alkaloid biosynthesis in root culture of Psychotria ipecacuanha. The Journal of Biological Chemistry, 285, $7722-7738$.

44. Park, S., Yu, M., \& Facchini, P. (2002). Antisense RNA-mediated suppression of benzophenanthridine alkaloid biosynthesis in transgenic cell cultures of California poppy. Plant Physiology, 128, 696-706.

45. Pauli, H., \& Kutchan, T.M. (1998). Molecular cloning and functional heterologous expression of two alleles encoding (S)- $N$-methylcoclaurine 3'-hydroxylase (CYP80B1), a new methyl jasmonate-inducible cytochrome P-450-dependent mono-oxygenase of benzylisoquinoline alkaloid biosynthesis. The Plant Journal, $13,793-801$.

46. Samanani, N., Liscombe, D.K., \& Facchini, P.J. (2004). Molecular cloning and characterization of norcoclaurine synthase, an enzyme catalyzing the first committed step in benzylisoquinoline alkaloid biosynthesis. The Plant Journal, 40, 302-313.

47. Sato, F. (2005). RNAi and functional genomics. Plant Biotechnology, 22, 431-442.

48. Sato, F., Hashimoto, T., Hachiya, A., Tamura, K.-i., Choi, K.-B., Morishige, T., et al. (2001). Metabolic engineering of plant alkaloid biosynthesis. Proceedings of the National Academy of Sciences of the United States of America, 98, 367-372.

49. Sato, F., Inai, K., \& Hashimoto, T. (2007a). Metabolic engineering in alkaloid biosynthesis: case study in tyrosine- and putrescine-derived alkaloids. In R. Verpoorte, A.W. Alfermann, \& T.S. Johnson, (Eds.). Applications of Plant Metabolic Engineering (pp.145-173). Dordrecht: Springer.

50. Sato, F., Inui, T., \& Takemura, T. (2007b). Metabolic engineering in isoquinoline alkaloid biosynthesis. Current Pharmaceutical Biotechnology, 8, 211-218.

51. Sato, F., \& Yamada, Y. (2008). Engineering formation of medicinal compounds in cell cultures. In H.J. Bohnert, H. Nguyen, \& N.G. Lewis (Eds), Advances in Plant Biochemistry and Molecular Biology, Vol. 1. (pp. 311-345), Amsterdam: Elsevier.

52. Takemura, T., Chow, Y-L., Todokoro, T., Okamoto, T., \& Sato, F. (2010a). Overexpression of rate-limiting enzymes to improve alkaloid productivity. In J.M. Walker (Series Ed.), \& A.G. Fett-Neto (Vol. Ed.), Methods in Molecular Biology, Vol. 643. Plant Secondary Metabolism Engineering: Methods and Protocols (pp. 
33-45), New York: Springer.

53. Takemura, T., Ikezawa, N., Iwasa, K., \& Sato, F. (2010b). Metabolic diversification of benzylisoquinoline alkaloid biosynthesis through the introduction of a branch pathway in Eschscholzia californica. Plant and Cell Physiology, 51, 949-959.

54. Takemura, T., Ikezawa, N., Iwasa, K., \& Sato, F. (2012). Molecular cloning and characterization of a cytochrome $\mathrm{P} 450$ in sanguinarine biosynthesis from Eschscholzia californica cells. Phytochemistry, 91, 100-108.

55. Takeshita, N., Fujiwara, H., Mimura, H., Fitchen, J., Yamada, Y., \& Sato, F. (1995). Molecular cloning and characterization of $S$-adenosyl-L-methionine: scoulerine-9-O-methyltransferase from cultured cells of Coptis japonica. Plant and Cell Physiology, 36, 29-36.

56. Unterlinner, B., Lenz, R., \& Kutchan, T.M. (1999). Molecular cloning and functional expression of codeinone reductase: the penultimate enzyme in morphine biosynthesis in the opium poppy Papaver somniferum. The Plant Journal, 18(5), 465-475.

57. Verpoorte, R., van Der Heijden, R., van Gulik, W.M., \& Ten Hoopen, H.J.G. (1991). Plant biotechnology for the production of alkaloids: present status and prospects. In A. Brossi (Ed), The Alkaloids: Chemistry and Pharmacology, Vol. 40. (pp. 1-187), New York: Academic Press.

58. Vogel, M., Lawson, M., Sippl, W., Conarad, U., \& Roos, W. (2010). Structure and mechanism of sanguinarine reductase, an enzyme of alkaloid detoxification. The Journal of Biological Chemistry, 285, 18397-18406.

59. Wallner, S., Winkler, A., Riedl, S., Dully, C, Horvath, S., Gruber, K. et al. (2012). Catalytic and structural role of a conserved active site histidine in berberine bridge enzyme. Biochemistry, 51, 6139-6147.

60. Winkler, A., Motz, K., Riedl, S., Puhl, M., Macheroux, P., \& Gruber, K. (2009). Structural and mechanistic studies reveal the functional role of bicovalent flavinylation in berberine bridge enzyme. The Journal of Biological Chemistry, 284, 19993-20001.

61. Winzer, T., Gazda, V., He, Z., Kaminski, F., Kern, M., Larson, T.R. et al. (2012). A Papaver somniferum 10-gene cluster for synthesis of the anticancer alkaloid noscapine. Science, 336, 1704-1708.

62. Yamada, Y., Kokabu, Y., Chaki, K., Yoshimoto, T., Ohgaki, M., Yoshida, S., et al. (2011a). Isoquinoline alkaloid biosynthesis is regulated by a unique bHLH-type transcription factor in Coptis japonica. Plant and Cell Physiology, 52, 1131-1141.

63. Yamada, Y., Koyama, T., \& Sato, F. (2011b). Basic helix-loop-helix transcription 
factors and regulation of alkaloid biosynthesis. Plant Signaling \& Behavior, 6, $1627-1630$.

64. Yamada, Y., \& Sato, F. (2013). Transcription factors in alkaloid biosynthesis. International Review of Cell \& Molecular Biology, 305, 339-382.

65. Zhang, Y., Zhang, F., Li, X., Baller, J.A., Qi, Y., Starker, C.G. et al. (2013). Transcription activator-like effector nucleases enable efficient plant genome engineering. Plant Physiology, 161, 20-27.

66. Ziegler, J., Voigtlaender, S., Schmidt, J., Kramell, R., Miersch, O., Ammer, C. et al. (2006). Comparative transcript and alkaloid profiling in Papaver species identifies a short chain dehydrogenase/reductase involved in morphine biosynthesis. The Plant Journal, 48, 177-192. 
Figure 1. Main pathway in isoquinoline alkaloid biosynthesis. Major isoquinoline alkaloids, such as aporphine-, benzophenanthridine- and protoberberine-type isoquinoline alkaloids, are synthesized through a common pathway derived from norcoclaurine to reticuline. Aporphine alkaloids are synthesized from $(S)$-reticuline by the reaction of corytuberine synthase (CYP80G2). Protoberberine and benzophenanthridine alkaloids are synthesized from $(S)$-scoulerine produced from $(S)$-reticuline by the reaction of berberine bridge enzyme (BBE). Ipecac and some other IQAs are synthesized from dopamine without norcoclaurine biosynthesis. The names of the biosynthetic enzymes for which cDNA has been isolated and characterized are shown. Abbreviations for biosynthetic enzymes are defined in the text, except for TYDC (tyrosine/DOPA decarboxylase; Facchini et al., 2000) and TyrAT (tyrosine aminotransferase; Lee \& Facchini, 2011).

Figure 2.

Extended pathways in isoquinoline alkaloid biosynthesis; from reticuline to protoberberine, benzophenanthridine, morphinan and noscarpine alkaloids (a) Protoberberine and benzophenanthridine alkaloid biosynthesis has mainly been characterized in Coptis japonica $(C j)$ and Eschscholzia californica (Es) cells, while some genes have been isolated from Papaver somniferum (Ps). (b) Morphinan and noscarpine biosynthesis were characterized in Papaver somniferum.

The names of the biosynthetic enzymes for which cDNA has been isolated and characterized are shown. Abbreviations for biosynthetic enzymes are defined in the text, except for CoOMT (columbamine $O$-methyltransferase), and 7OMT (reticuline 7-O-methyltransferase).

Figure 3.

Ipecac OMT alignment and reaction specificity

Figure 4.

Yield improvement by the ectopic expression of $C$. japonica $(C j)$ 6OMT in California poppy cells.

Figure 5.

Metabolic diversification by the introduction of a branch pathway

The introduction of a novel branch pathway in California poppy induced the production of uncommon protoberberine-type alkaloids, whereas major products were further 
metabolized to di-methoxy-type benzophenanthridine alkaloids by endogenous enzymes.

Figure 6.

Quality modification by the trimming of a pathway

Knockdown of BBE expression with double-stranded RNA strongly reduced endogenous alkaloid accumulation and increased the accumulation of a precursor, reticuline, and some metabolites such as 7-O-methylreticuline. 

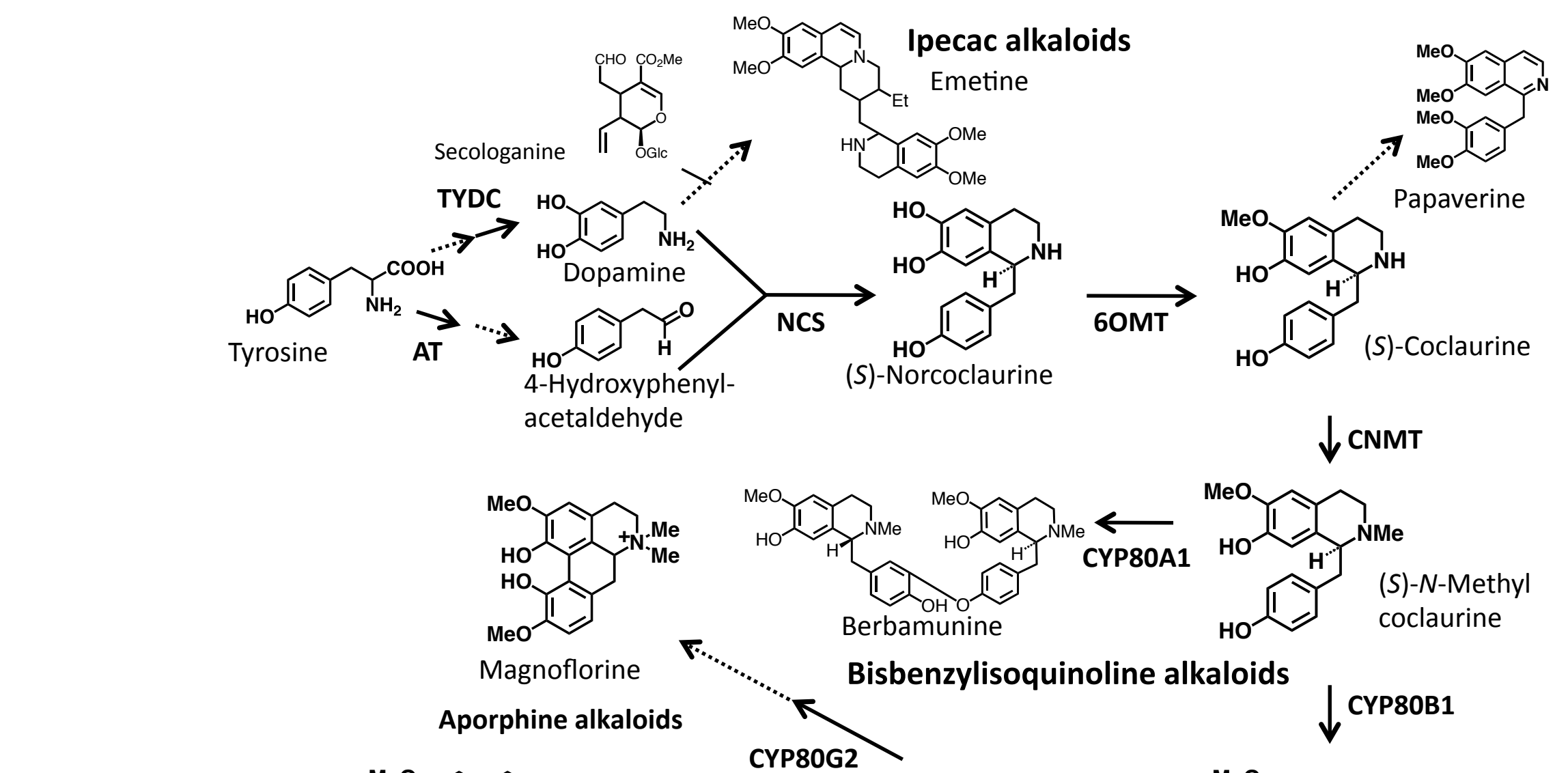

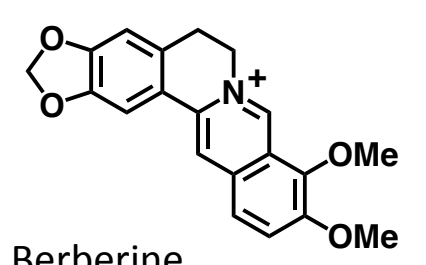

Protoberberine alkaloids

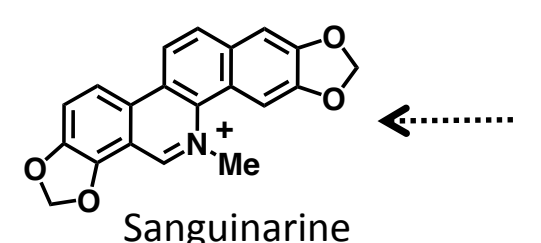

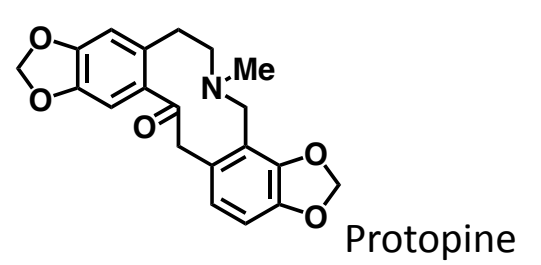

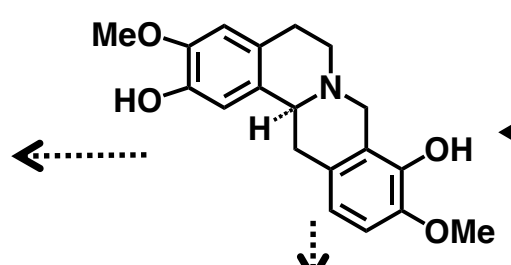

(S)-Scoulerine

BBE

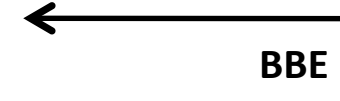

Protopine

Benzophenanthridine alkaloids

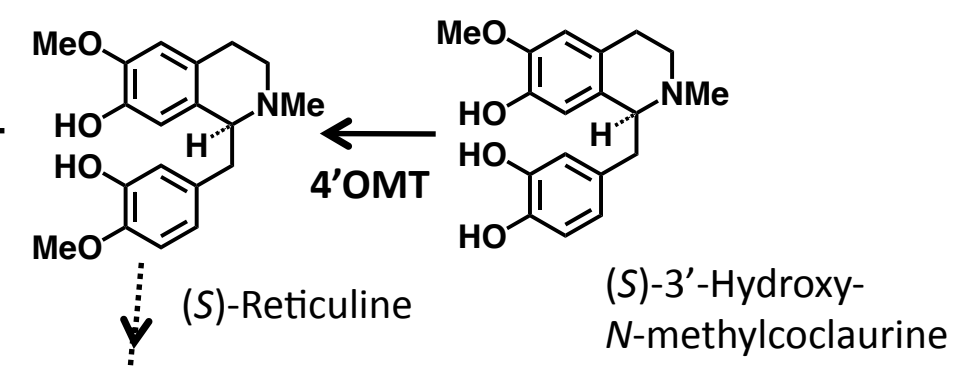

Fig. 1 


\section{Protoberberine pathway}

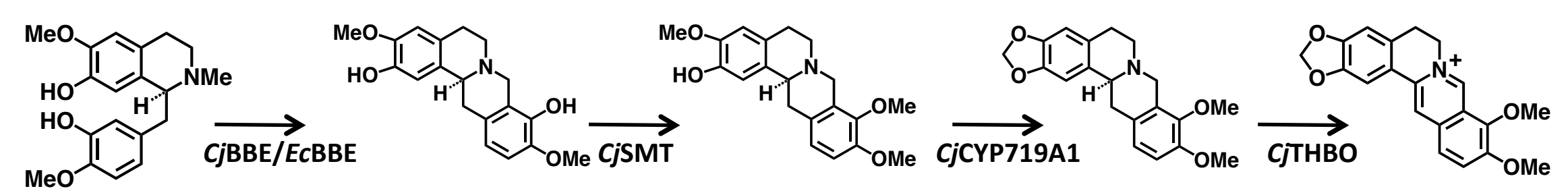

(S)-Reticuline

(S)-Scoulerine

(S)-Tetrahydrocolumbamine

(S)-Tetrahydroberberine

Berberine

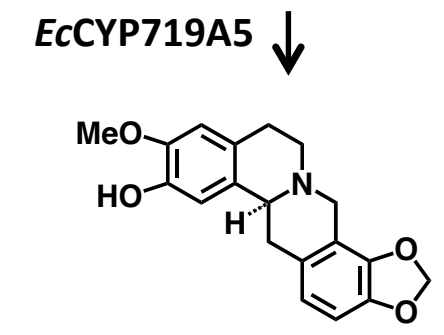

EcCYP719A2/A3 $\downarrow$ Cheilanthifoline

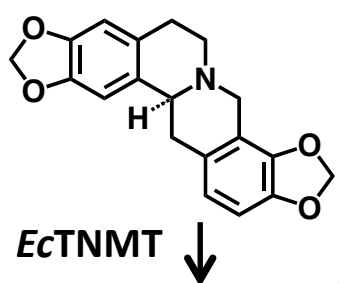

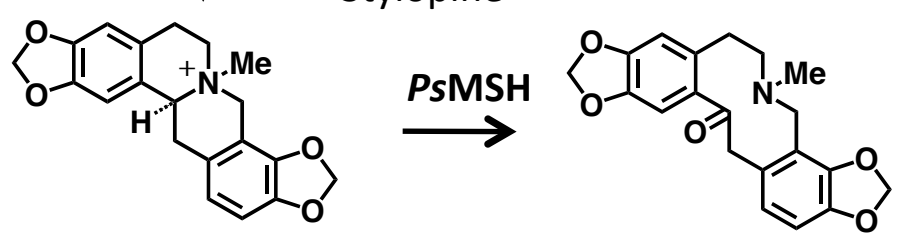

$N$-Methylstylopine

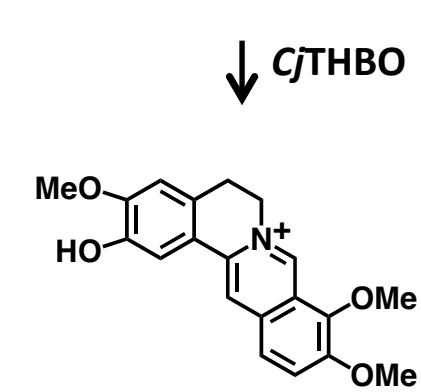

Columbamine
CjCoOMT

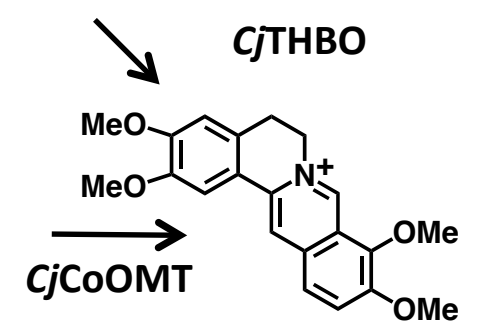

Palmatine

\section{Benzophenanthridine pathway}

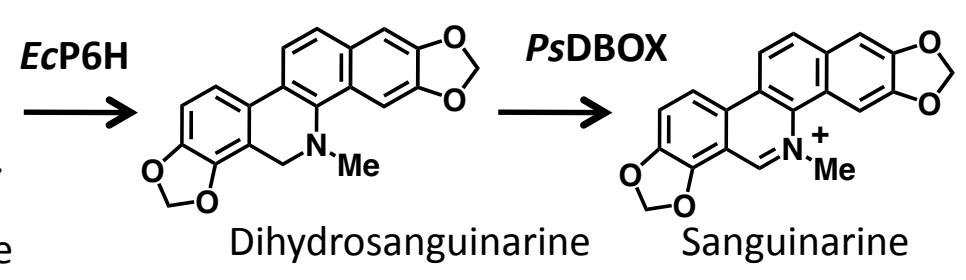

Fig. $2(A)$ 

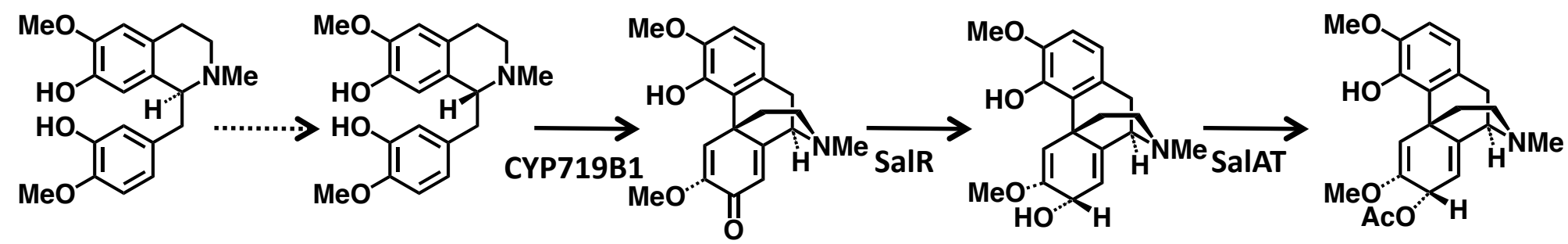

(S)-Reticuline

(R)-Reticuline

Salutaridine

Salutaridinol

Salutaridinol-7-O-acetate

BBE $\downarrow$
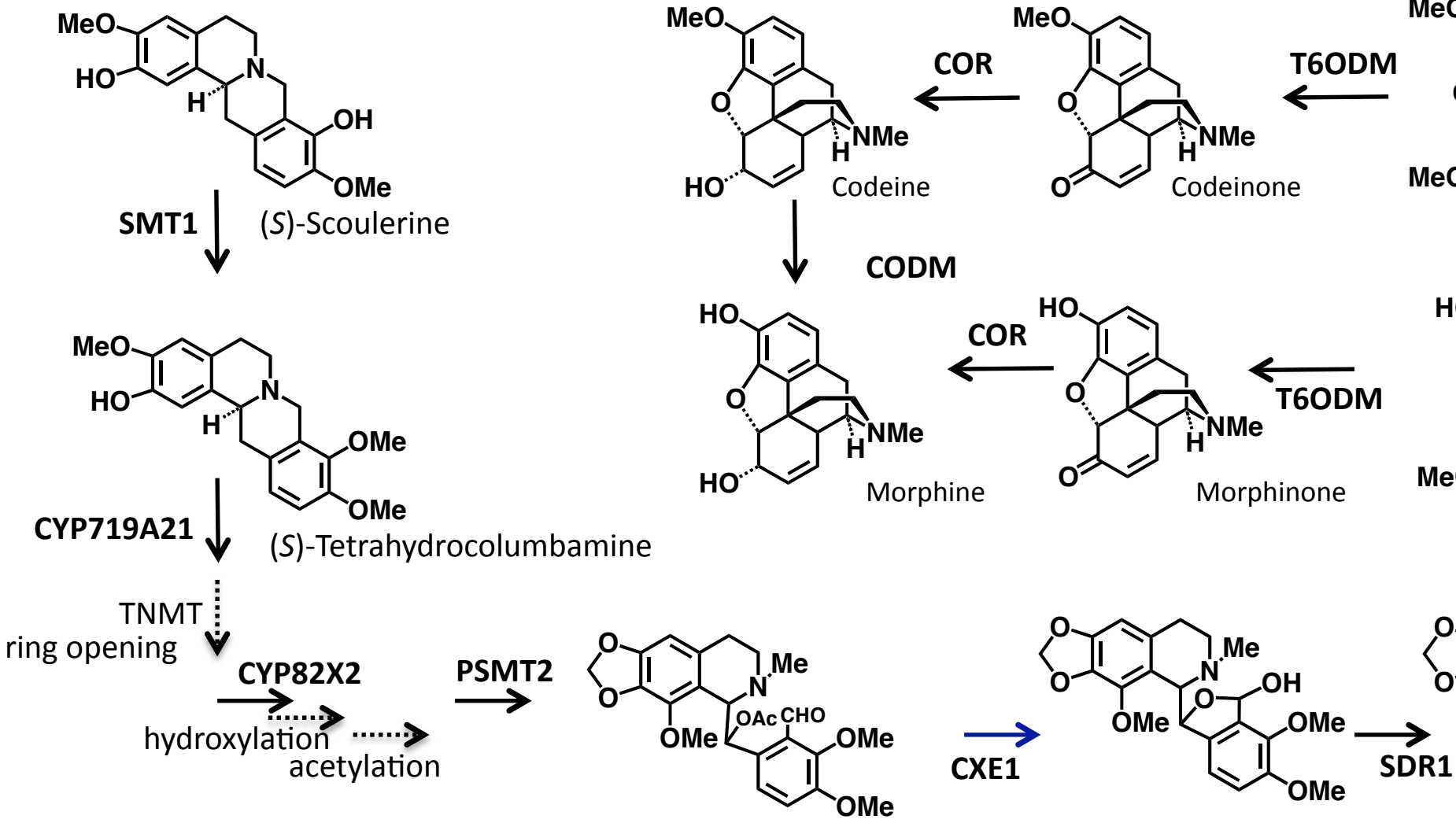

Noscapine pathway

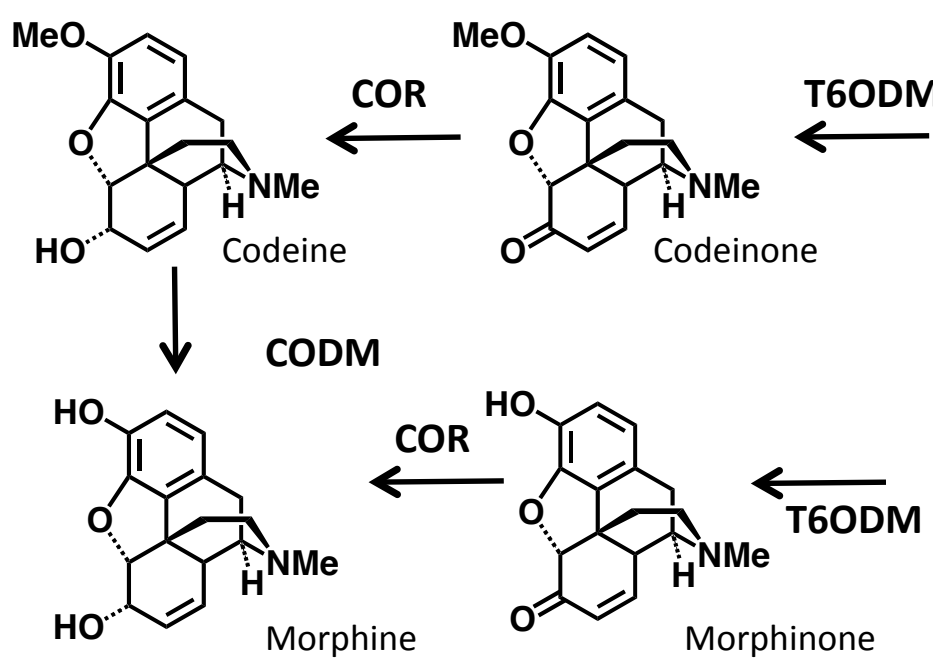

Spontaneous

\section{Morphinan pathway}
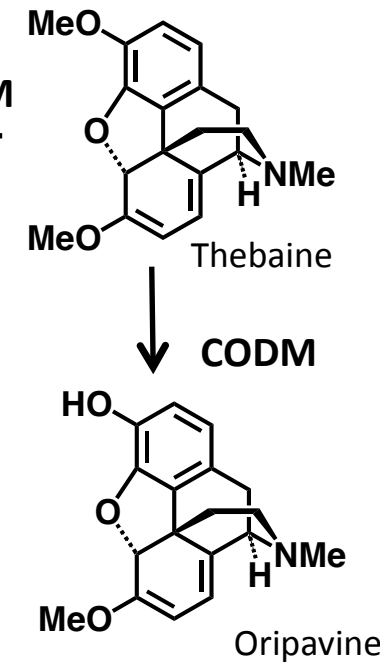

Oripavine

Papaveroxine narcotinehemiacetal

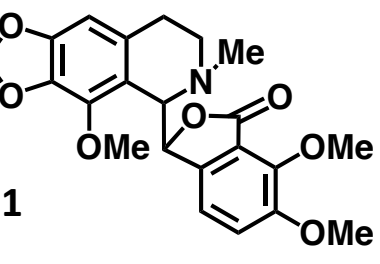

noscapine

Fig. 2 (B) 


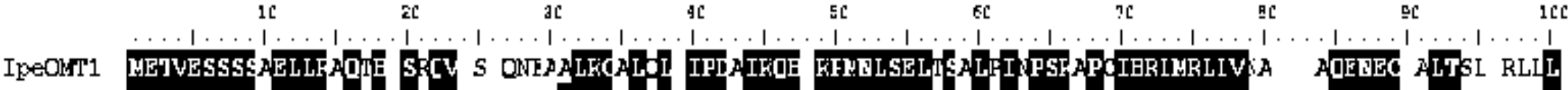

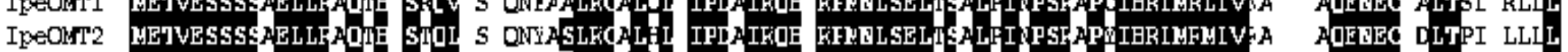

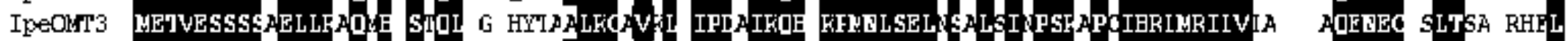

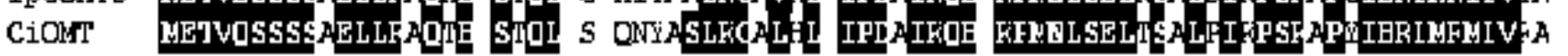

AOEEEC DLTPI LLLL

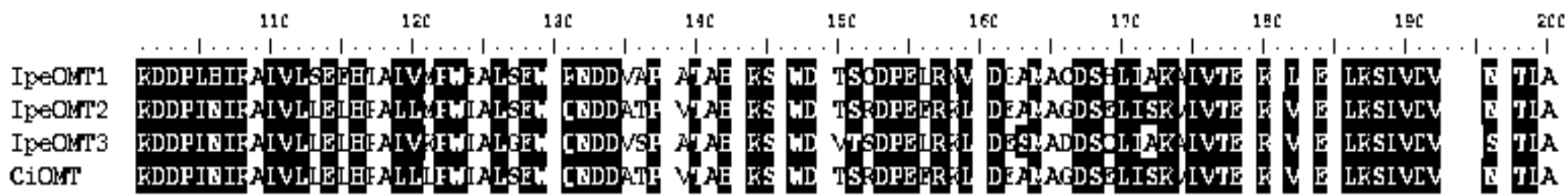

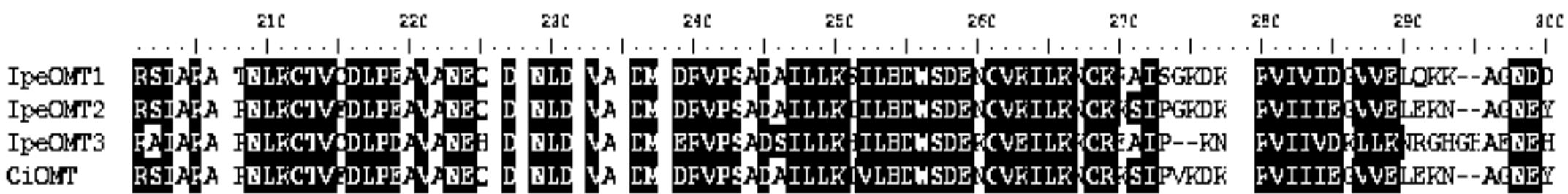

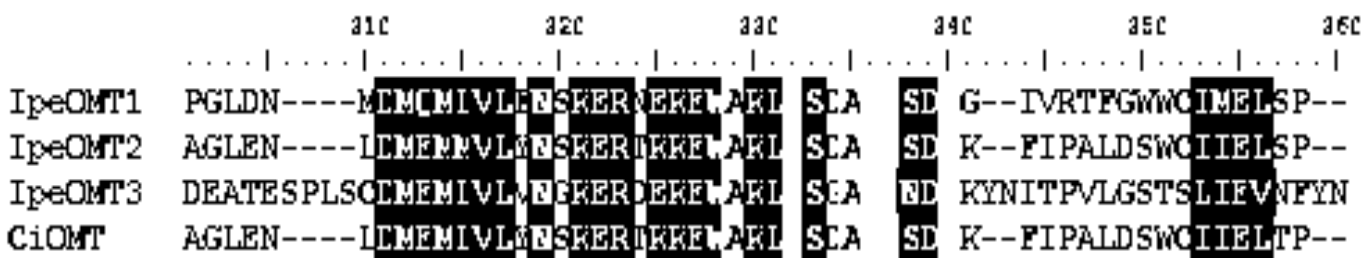<smiles>CCC1CN2C=Cc3cc(OC)c(OC)cc3C2CC1CC1NCCc2cc(O)c(O)cc21</smiles>

7'-O-Demethylcephaeline

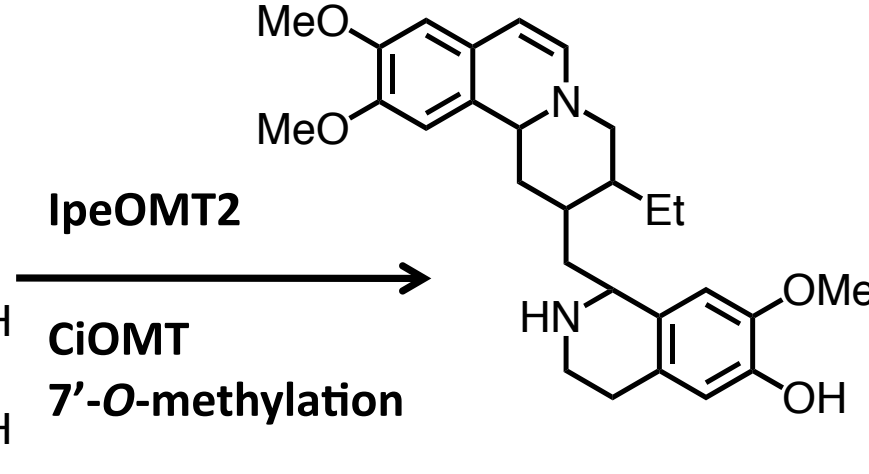

Cephaeline

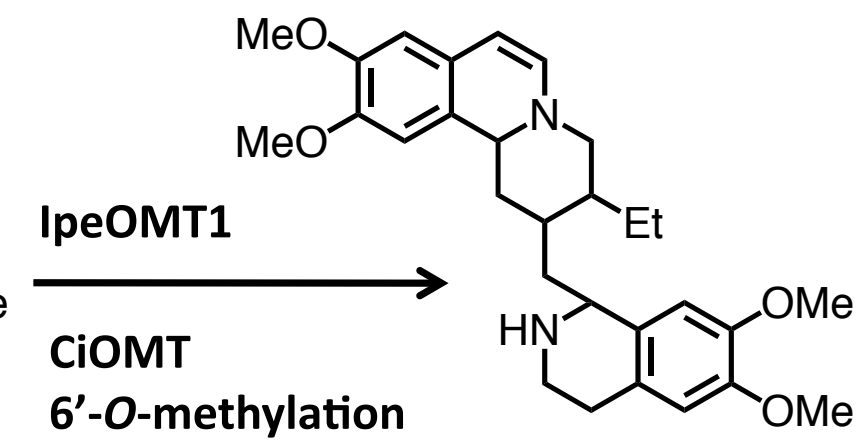

Emetine

Fig. 3 

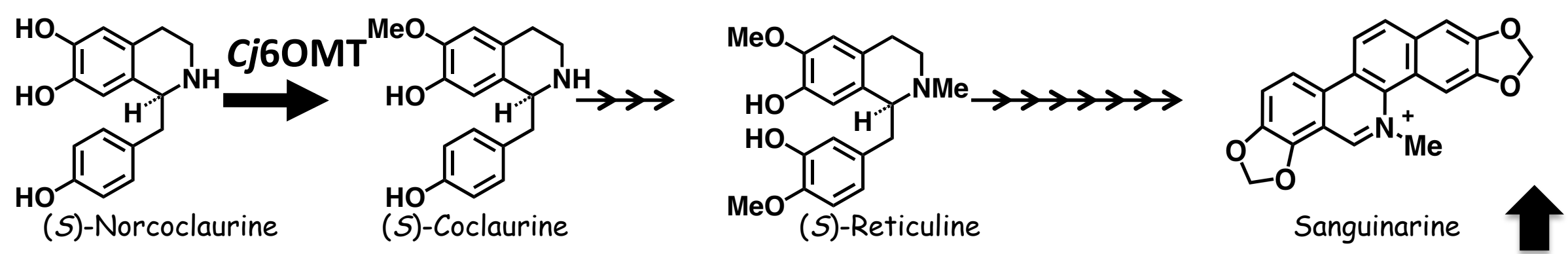

Figure 4 


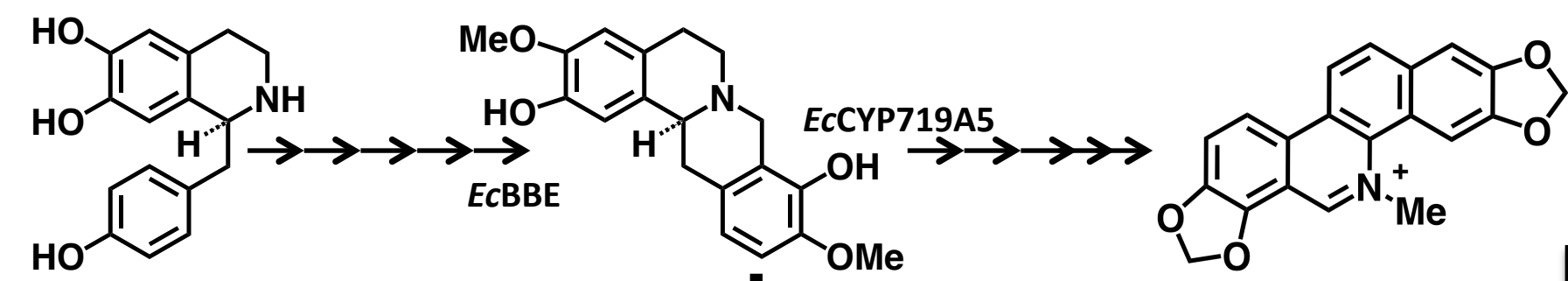

(S)-Norcoclaurine

Sanguinarine

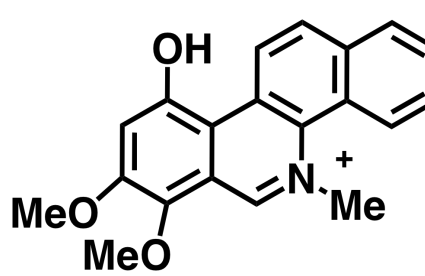

10-Hydroxychelerythrine

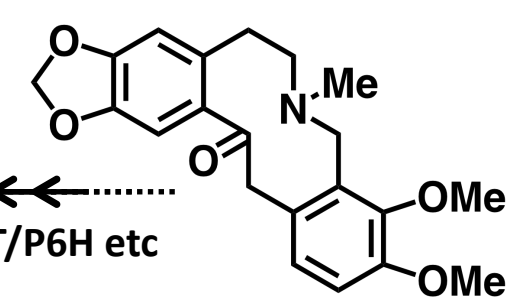

Allocryptopine
(S)-Scoureline

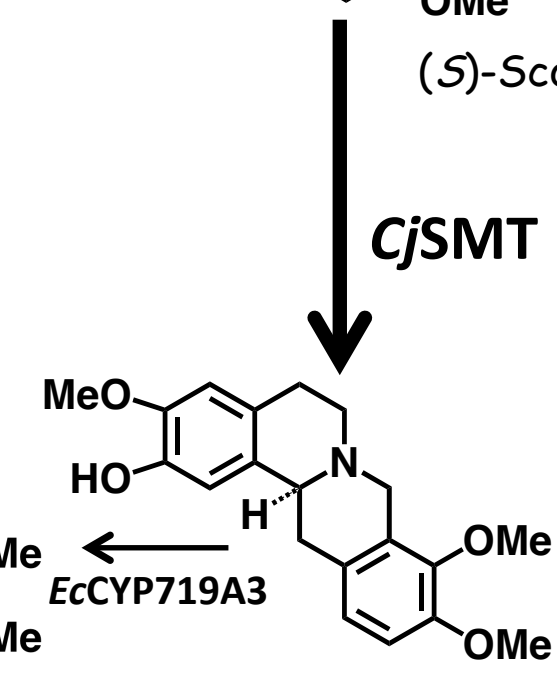

(S)-Tetrahydrocolumbamine

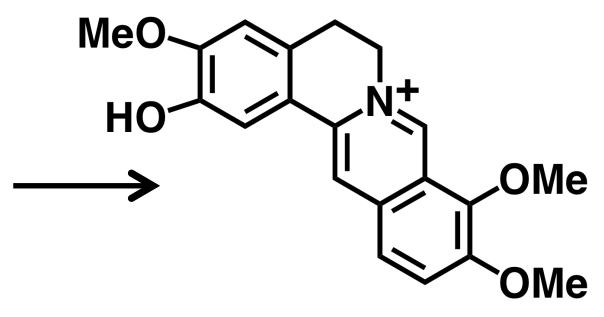

Columbamine

Figure 5 

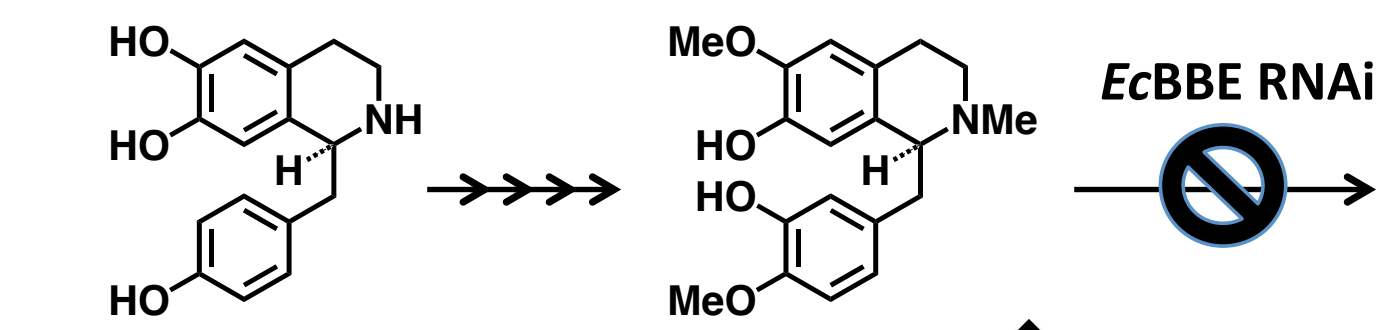

(S)-Norcoclaurine

(S)-Reticuline

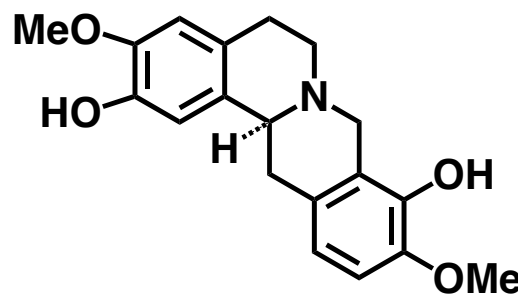

(S)-Scoulerine

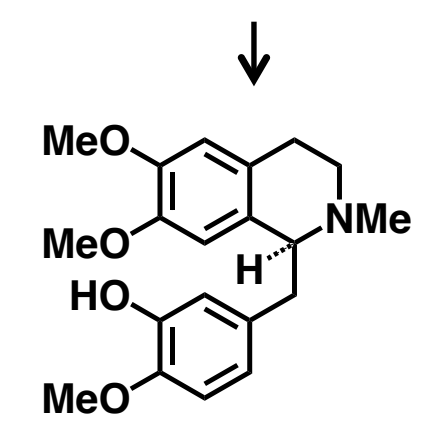

(S)-7-O-Methylreticuline

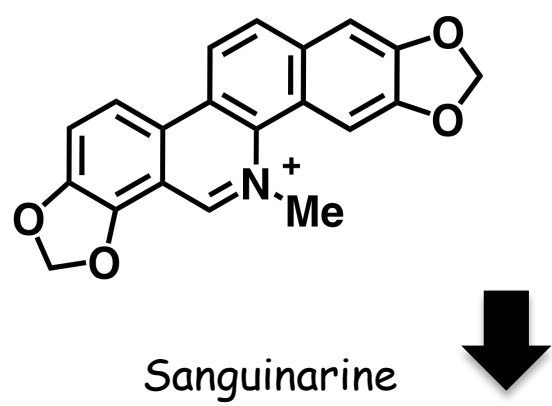

Figure 6 